\title{
Nerve conduction studies, skeletal muscle EMG, and sphincter EMG in multiple system atrophy
}

\author{
P P Pramstaller, G K Wenning, S J M Smith, R O Beck, N P Quinn, C J Fowler
}

\begin{abstract}
Although autonomic failure, parkinsonism, and cerebellar and pyramidal signs are well documented in multiple system atrophy, much less is known about the frequency and severity of involvement of the peripheral nervous system. The frequency and nature of peripheral nerve involvement has therefore been determined in 74 patients with multiple system atrophy using nerve conduction studies and skeletal muscle EMG. These findings were compared with those on sphincter EMG. Ninety per cent of the patients had an abnormal sphincter EMG, indicating denervation and reinnervation consistent with anterior horn cell loss in Onuf's nucleus, but only $40 \%$ had either abnormal nerve conduction studies (mixed sensorimotor axonal neuropathy in $17 \cdot 5 \%$ ) or abnormal skeletal muscle EMG (suggesting partial denervation in $22.5 \%$ ).

These data indicate a remarkable selective vulnerability of the anterior horn cells of Onuf's nucleus innervating external sphincter muscles relative to those supplying skeletal muscle in patients with multiple system atrophy. If this selective pattern of involvement can be explained it may be a clue to pathogenetic mechanisms in multiple system atrophy.
\end{abstract}

(F Neurol Neurosurg Psychiatry 1995;58:618-621)

Department of Clinical Neurology P P Pramstaller G K Wenning

N P Quinn

Department of Clinical

Neurophysiology

S J M Smith

Department of

Uro-Neurology,

Institute of Neurology,

Queen Square,

London, UK

R O Beck

C J Fowler

Correspondence to:

$\operatorname{Dr} N \mathrm{P}$ Quinn, Institute of Neurology, National Hospital for Neurology and Neurosurgery, Queen

Square, London WC1N 3BG, UK.

Received 8 September 1994 and in revised form 4 January 1995 Accepted 6 January 1995
PATIENTS

Seventy four patients with clinically probable multiple system atrophy (pathologically established in 11) underwent peripheral electrophysiological studies $(n=40)$ or sphincter EMG ( $n=71)$, or both. All patients undergoing peripheral electrophysiological studies had both nerve conduction studies and skeletal muscle EMG. In 35 patients both peripheral electrophysiological studies and sphincter EMG were performed. Fifty one of the patients were men $(68 \%)$ and 23 were women $(32 \%)$. Their mean age at the time of sphincter electromyography was 52.8 (range 33-67) years, and at the time of peripheral electrophysiological studies (nerve conduction studies and skeletal muscle EMG) it was 55.1 (41-67) years.

Patients were diagnosed according to published clinical criteria. ${ }^{10}$ There were $56(76 \%)$ patients with multiple system atrophy of striatonigral degeneration (SND) type, either with (24) or without (32) cerebellar signs. Eighteen $(24 \%)$ patients were classified as having multiple system atrophy of olivopontocerebellar atrophy (OPCA) type, either without ("pure" OPCA type, eight) or with ("predominant" OPCA type, 10) extrapyramidal features.

The mean age at onset of the first symptoms was 50.3 (range 33-71) years. Symptoms at onset were autonomic dysfunction in $28(38 \%)$, parkinsonism in $25(34 \%)$, cerebellar dysfunction in seven $(9 \%)$, and mixed in the remaining $18 \%$ of these patients. The most common initial diagnosis was Parkinson's disease, then cerebellar syndrome, and then multiple system atrophy.

At the time of investigation autonomic dysfunction was present in $65(88 \%)$ of the 74 patients. The mean age at onset for autonomic dysfunction was $50 \cdot 1$ (range 38-71) years. Micturition dysfunction was present in all of these 65 patients (frequency in 47, urgency in 51 , incontinence in 42 , retention in 18, and nocturia in 44). Erectile failure was present in $47(92 \%)$ of the 51 male patients. Symptomatic orthostatic hypotension was present in 40 (54\%) patients with autonomic dysfunction, but recurrent syncopal attacks had developed only in six (15\%).

Parkinsonism developed in $56(76 \%)$ patients at mean age $53 \cdot 1$ (range 34-73) years, cerebellar signs developed in 34 (46\%) at mean age $57 \cdot 7$ (range $38-71$ ) years, and pyramidal signs in $39(53 \%)$ patients, first 
noted at mean age $57 \cdot 3$ (range $36-67$ ) years.

Lower motor neuron signs with reduced $(n=1)$ or absent $(n=3)$ reflexes and muscle wasting $(n=4)$ were reported in four $(5 \%)$ patients, and fasciculations were found in one. Sensory disturbances were present in 21 $(28 \%)$ patients (paraesthesiae, 13; loss of vibration sense, nine; impaired sensation of light touch and pinprick, two; and burning pain one).

Respiratory stridor was present in 22 $(30 \%)$ of the 74 patients, four of whom underwent tracheostomy. Mean age at onset of this symptom was 52.9 (range 39-67) years.

\section{NERVE CONDUCTION STUDIES}

Standard techniques were used to measure peripheral sensory and motor nerve conduction. ${ }^{11}$ Sensory action potentials of the median, ulnar, and sural nerves were recorded using skin electrodes; compound muscle action potentials and motor conduction velocities were measured in the arm (median or ulnar nerve) and leg (posterior tibial nerve or common peroneal nerve) after correcting for limb temperature. Measurements were compared with age matched normative data established at our laboratory.

\section{SKELETAL MUSCLE ELECTROMYOGRAPHY}

Skeletal muscle EMG was performed with concentric needle electrodes. Muscles were examined for spontaneous activity (fibrillations, positive sharp waves, fasciculations) and motor unit potentials (interference pattern, morphology, and size of motor unit potentials).

\section{SPHINCTER ELECTROMYOGRAPHY}

A concentric needle electrode was used and individual motor units from the urethral sphincter, the anal sphincter, or both were recorded and analysed on a Dantec Counterpoint. ${ }^{12}$ In each patient 10 different motor units were captured with a trigger delay line and two parameters of abnormality analysed as described by Eardley et al. ${ }^{13}$ Both mean duration of 10 individual motor units, and the proportion of highly abnormal motor units (>10 ms) were calculated.

\section{STATISTICAL ANALYSIS}

Statistical analysis was performed by $\chi^{2}$ test.

\section{Results}

PERIPHERAL ELECTROPHYSIOLOGICAL STUDIES Sixteen $(40 \%)$ of the 40 patients who underwent peripheral electrophysiological studies had pathological results on either nerve conduction studies or skeletal muscle EMG. These investigations gave normal results in 33 $(82.5 \%)$ and $31(77 \cdot 5 \%)$ respectively of the patients studied.

Nerve conduction studies-Abnormal nerve conduction was found in seven patients. Evidence of mixed sensorimotor axonal neuropathy was seen in three patients (generalised reduction or absence of sensory action potentials and reduced amplitude of compound muscle action potentials in arms and legs). In three further patients sensory action potentials and compound muscle action potentials in the legs were small or absent. In one patient, the abnormality was confined to an absent sural sensory action potential. One further case had an ulnar nerve lesion; no other entrapment neuropathies were seen.

Skeletal muscle electromyography-Abnormalities suggesting partial denervation were found in nine patients (fibrillations and fasciculations, five; polyphasic compound muscle action potentials, nine; and large motor units, two). In all nine patients the changes were more pronounced distally. None of these nine patients had evidence of a polyneuropathy.

\section{SPHINCTER ELECTROMYOGRAPHY}

Sixty four out of $71(90 \%)$ patients investigated had an abnormal sphincter EMG consistent with denervation and subsequent chronic re-innervation in the sphincter muscles. These findings, in the absence of pelvic nerve or cauda equina lesion or any clear clinical history suggesting damage to the peripheral innervation, are highly suggestive of anterior horn cell loss in Onuf's nucleus ${ }^{14}$ and help to confirm the diagnosis of multiple system atrophy in the appropriate clinical context. Anal sphincter EMG was technically easier and less uncomfortable for the patients than urethral sphincter EMG, and was equally satisfactory for diagnostic purposes. ${ }^{15}$ Urinary urgency or incontinence was present in all patients with pathological sphincter EMG. Of the four patients with normal sphincter EMG, only one had evidence of urinary urge incontinence at the time of investigation.

\section{PERIPHERAL ELECTROPHYSIOLOGICAL STUDIES} V SPHINCTER ELECTROMYOGRAPHY

The mean age at the time of pathological sphincter EMG was $52 \cdot 8$ (range 38-67) years, and mean duration between onset of micturition dysfunction and sphincter EMG was 3.7 (range $1-10$ ) years. The mean age at the time of pathological peripheral electrophysiological studies was 56.6 (range 49-67) years for the partial denervation group, and 49.8 (range 48-66) years for the neuropathy group). The comparison between the sensitivity of the two techniques for detecting abnormality has been biased against sphincter EMG, as this test was performed a mean of 3.8 years before the general neurophysiology. It could well be that if sphincter EMG had been performed when the patients were in a more advanced stage an even higher proportion of abnormalities might have been found.

Of the 35 patients who underwent both investigations, 31 (89\%) had an abnormal sphincter EMG. Twenty five $(81 \%)$ of these 31 had normal nerve conduction studies and 23 (74\%) normal skeletal muscle EMG. Among the four patients with normal sphincter EMG, nerve conduction studies were normal in all four, and skeletal muscle EMG 
abnormal in one. One or other peripheral electrophysiological study abnormality was therefore found in $14(45 \%)$ of these 31 patients. $\chi^{2}$ Tests showed no significant association between an abnormal sphincter EMG, and abnormal peripheral nerve conduction studies or skeletal muscle EMG.

Among the 22 patients with a history of respiratory stridor, $100 \%$ also had a pathological sphincter EMG.

\section{Discussion}

Our data emphasise the important diagnostic role of sphincter EMG by contrast with peripheral electrophysiological studies in multiple system atrophy. Thus $94 \%$ of the patients with multiple system atrophy in this study had an abnormal sphincter EMG with signs of denervation and re-innervation. The striated muscles of the urethral and anal sphincters are supplied by Onuf's nucleus, a slender longitudinal cell group extending from the middle of $S 2$ to the rostral one third of S3. ${ }^{16}$ Loss of these neurons is followed by re-innervation of target muscles, probably as a result of collateral sprouting of surviving motor neurons, analogous to that seen in skeletal muscle of patients with motor neuron disease. ${ }^{17}$ This results in prolonged, polyphasic potentials of increased amplitude. The external sphincter muscles differ from skeletal limb muscles in exhibiting tonic EMG activity, which persists even during sleep and general anaesthesia. ${ }^{18}$ The posterior cricoarytenoid muscle, supplied by the nucleus ambiguus, also shows similar tonic activity to maintain abductor tone. It is recognised that laryngeal stridor and defects of phonation are often seen in multiple system atrophy, and EMG studies of the posterior cricoarytenoid muscle have also shown unequivocal evidence of denervation in this condition, ${ }^{19}$ suggesting a common vulnerability.

Some investigators ${ }^{1-9}$ have suggested that both somatic anterior horn cells as well as peripheral nerves are commonly affected in multiple system atrophy, and their involvement has therefore been regarded as part of the clinical range of multiple system atrophy. Nerve conduction studies and skeletal muscle EMG were normal, however, in most of our patients.

Among the 35 patients who underwent both sphincter EMG and peripheral electrophysiological studies, sphincter EMG was abnormal in $89 \%$, but nerve conduction studies and skeletal muscle EMG were abnormal in only $17 \%$ and $26 \%$ respectively. This evidence of frequent involvement of motor units of external sphincter muscles, with relative preservation of skeletal muscle motor units, indicates selective vulnerability of the first. Although all patients in this series had symptoms of bladder dysfunction and most had abnormal sphincter EMG, it is not proposed that their incontinence is due to denervation of their sphincter. Other studies ${ }^{151720}$ have shown that detrusor hyperreflexia and incomplete bladder emptying are often found in patients with multiple system atrophy, and it is presumably these disorders that are more important in causing the early, severe incontinence so characteristic of the disease.

Anterior horn cells supplying skeletal musculature are of three distinct types in terms of cell body size, location, and function: large $\alpha$ motor neurons and medium sized $\gamma$ motor neurons mainly located in the lateral and medial nuclei, and small neurons distributed in the intermediate zone (dorsomedial part) of the spinal anterior horn. ${ }^{21}$ Distinct disease specific patterns of neuronal cell loss in degenerative anterior horn cell disease have been postulated by Terao et al. ${ }^{8}$ They found that in multiple system atrophy, by contrast with amyotrophic lateral sclerosis, small neurons in the intermediate zone are very depopulated, whereas large medium sized neurons of the medial and lateral nuclei tend to be preserved. The specific loss of these neurons, which seem to serve as interneurons, could play a part in the generation of muscular rigidity and possibly also of the pyramidal signs seen in patients with multiple system atrophy. ${ }^{8}$ The pathogenetic mechanism underlying selective anterior horn cell loss in Onuf's nucleus with relative preservation of other anterior horn cell groups, however, remains unknown.

We conclude that nerve conduction studies and skeletal muscle EMG are each normal in about three quarters of patients with multiple system atrophy. By contrast, sphincter EMG studies are frankly pathological in almost all patients, suggesting a differential, selective vulnerability of different anterior horn cell groups within the spinal cord.

1 Galassi G, Nemni R, Baraldi A, et al. Peripheral neuropathy in multiple system atrophy. Neurology 1982;32: 1116-21.

2 Singh N, Fahn S. Electrophysiological studies in the ShyDrager syndrome. Neurology 1980;30:394.

3 Toghi $\mathrm{H}$, Tabuchi $M$, Tomonaga $M$, et al. Selective loss of small myelinated and unmyelinated fibers in ShyDrager syndrome. Acta Neuropathol (Berl) 1982;57: 282-6.

4 Low PA, Thomas JE, Dyck PJ. The splanchnic autonomic outflow in Shy-Drager syndrome and orthostatic hypotension. Ann Neurol 1978;4:511-4.

5 Cohen J, Low P, Fealy R, et al. Somatic and autonomic function in progressive autonomic failure and multiple function in progressive autonomic failure and

6 Bannister R, Ellison M, Morgan-Hughes J. Amyotrophy in multiple system atrophy. In: Bannister $R$, ed. Autonomic failure. 2nd ed. Oxford: Oxford Medical Publishers, 1988:559-68.

7 Montagna P, Martinelli P, Rizzuto N, et al. Amyotrophy in Shy-Drager syndrome. Acta Neurol Belg 1983;83: 142-57

8 Terao S, Sobue G, Hashizume Y, et al. Disease specific pattern of neuronal cell loss in the ventral horn in amytrophic lateral sclerosis, multiple system atrophy, and $\mathrm{x}$ linked bulbospinal neuronopathy, with special reference to loss of small neurons in the intermediate zone. f Neurol 1994;241:196-203.

9 Sobue G, Terao S, Kachi T, et al. Somatic efferents in multiple system atrophy with autonomic failure: a clinimultiple system atrophy with autonomic failure: a cling

10 Quinn NP. Multiple system atrophy. In: Marsden CD, Fahn S, eds. Movement disorders 3. London: ButterFahn S, eds. Movem
worths, 1994:262-81.

11 Kimura J. Electrodiagnosis in diseases of nerve and muscle: principles and practice. Philadelphia: FA Davis, muscle: principle

12 Fowler CJ, Kirby RS, Harrison MJG, Milroy EJG, Turner-Warwick R. Individual motor unit analysis in the diagnosis of disorders of urethral sphincter innervation. F Neurol Neurosurg Psychiatry 1984;47:637-41. 
13 Eardley I, Quinn NP, Fowler CJ, et al. The value of urethral sohincter muscle electromyography in the differen-

14 Onufrowicz B. On the arrangement and function of the cell groups of the sacral region of the spinal cord in man. Archives of Neurology and Psychopathology 1900;3: 387-412.

15 Beck RO, Betts CD, Fowler CJ. Genitourinary dysfunction in multiple system atrophy: clinical features and treatment in 62 cases. 7 Urol 1994;151:1336-41.

16 Konno H, Yamamoto T, Iwasaki Y, et al. Shy-Drager syndrome and amyotrophic lateral sclerosis Cytoarchitectonic and morphometric studies of sacral autonomic neurons. $\mathcal{F}$ Neurol Sci 1986;73:193-204.

17 Swash M. Vulnerability of lower brachial myotomes in motor neuron disease: a clinical and single fibre EMG study. 7 Neurol Sci 1980;47:59-68

study. F Neurol Sci 1980;47:59-68.
18 Kirby R, Fowler CJ, Gosling J, et al. Urethro-vesical dysfunction in progressive autonomic failure with multiple system atrophy. $\mathcal{F}$ Neurol Neurosurg Psychiatry 1986;49: $554-62$.

19 Guindi GM, Bannister R, Gibson WPR, et al. Laryngea electromyography in multiple system atrophy with autonomic failure. If Neurol Neurosurg Psychiatry 1981;44: 49-53.

20 Lockhard JL, Webster GD, Sheremata W, et al. Neurogenic bladder dysfunction in the Shy-Drager syndrome. $f$ Urol 1981;126:119.

21 Rexed B. The cytoarchitectonic organisation of the spinal cord in cat. $\mathcal{F}$ Comp Neurol 1952;96:415-96. 